\title{
Modeling nasal physiology changes due to septal perforations
}

\author{
Daniel E. Cannon, MD, Dennis O. Frank, PhD, Julia S. Kimbell, PhD, David M. Poetker, MD, \\ MA, and John S. Rhee, MD, MPH \\ Departments of Otolaryngology and Communication Sciences, Medical College of Wisconsin, \\ Milwaukee, Wisconsin (Cannon, Poetker, Rhee) and Otolaryngology-Head and Neck Surgery, \\ University of North Carolina School of Medicine, Chapel Hill, North Carolina (Frank, Kimbell)
}

\begin{abstract}
Objective-To use computational fluid dynamics (CFD) technology to help providers understand 1) how septal perforations may alter nasal physiology and 2) how these alterations are influenced by perforation size and location.
\end{abstract}

Study Design-Computer simulation study

Setting-Facial Plastic and Reconstructive Surgery clinic

Subjects and Methods-With the aid of medical imaging and modeling software, septal perforations of 1 and $2 \mathrm{~cm}$ in anterior, posterior, and superior locations were virtually created in a nasal cavity digital model. CFD techniques were used to analyze airflow, nasal resistance, air conditioning, and wall shear stress.

Results-Bilateral nasal resistance was not significantly altered by a septal perforation. Airflow allocation changed, with more air flowing through the lower-resistance nasal cavity. This effect was greater for anterior and posterior perforations than for the superior location. At the perforation sites, there was less localized heat and moisture flux and wall shear stress in superior perforations compared to those in anterior or posterior locations. For anterior perforations, a larger size produced higher wall shear and velocity, while in posterior perforations a smaller size produced higher wall shear and velocity.

Conclusions-Septal perforations may alter nasal physiology. In the subject studied, airflow allocation to each side was changed as air was shunted through the perforation to the lowerresistance nasal cavity. Anterior and posterior perforations caused larger effects than those in a superior location. Increasing the size of anterior perforations and decreasing the size of posterior perforations enhanced alterations in wall shear and velocity at the perforation.

\section{Keywords}

Computer modeling; Septal perforation; Nasal surgery

Corresponding author: Daniel E. Cannon, MD, Department of Otolaryngology and Communication Sciences, Medical College of Wisconsin, 9200 W. Wisconsin Ave., Milwaukee, WI 53226, dcannon@ mcw.edu, (414) 805-5609.

Presented at the American Academy of Otolaryngology-Head and Neck Surgery annual meeting on September 10, 2012 in Washington, D.C.

Financial disclosures/conflicts of interest:

The authors have nothing to disclose. 


\section{Introduction}

Septal perforations can be frustrating for patients and challenging for nasal surgeons, as repair can be difficult. They exhibit an estimated overall prevalence of less than $1 \%$ in adults ${ }^{1}$ and can be idiopathic or occur from a variety of causes, including iatrogenic. Procedures with septal perforation as a potential complication include septoplasty, intranasal cautery, nasal packing, turbinate reduction, rhinoplasty, and sinus surgery. Other common causes are external nasal trauma resulting in septal fracture with development of a hematoma or repeated mucosal trauma from nose picking, nasal sprays, chemical irritants, or cocaine use. Finally, perforations can result from infectious, inflammatory, or neoplastic diseases $^{2}$.

Almost $40 \%$ of septal perforations are completely asymptomatic ${ }^{2}$. In other cases, perforations can be associated with crusting, bleeding, nasal obstruction, whistling, and pain. In particular, larger and more anterior perforations are more likely to produce symptoms ${ }^{3}$. Interestingly, there are surgeries, such as frontal sinus procedures and trans-sphenoidal approaches to the pituitary, which involve resection of portions of the nasal septum with no apparent symptomatic consequences to the patient. It is thought that disturbed airflow leading to mucosal drying is the cause of crusting and bleeding in septal perforations, ${ }^{4,5}$. However, the reason that certain perforations are asymptomatic is unclear. A previous study using a nasal model, Mink's boxes, suggested that the location of a septal perforation had no impact on flow pattern, as simulated perforations in both anterior and posterior locations exhibited turbulent flow ${ }^{5}$. These findings suggest that alterations in airflow patterns alone cannot be the sole explanation for symptom production in septal perforations.

Computational Fluid Dynamics (CFD) technology has emerged as a valuable tool for studying nasal physiology. CFD uses 3-dimensional (3-D) reconstructions of the nasal cavity created from computed tomography (CT) or magnetic resonance imaging (MRI) scans to simulate airflow patterns and velocity, wall shear, heat and moisture exchange, and other important aspects of nasal physiology. Prior CFD studies examining septal perforations have demonstrated changes in velocity, flow pattern, temperature distribution, and wall shear stress ${ }^{6-8}$. The combination of these factors has been proposed as the cause for production of symptoms. It was also demonstrated that the alterations were greater in larger perforations ${ }^{6}$.

However, these CFD studies have only examined perforations in the anterior region of the septum. The purpose of this study is to investigate the influence that size and location of a septal perforation have on airflow and velocity, wall shear stress, heat exchange, and humidification using CFD technology and discuss whether these effects may contribute to known patterns of symptomatology in patients with septal perforations.

\section{Methods}

\section{Patient Recruitment and Treatment}

Research methods were approved by the Institutional Review board at the Medical College of Wisconsin and written informed consent was obtained. This was a sub-project of a larger ongoing study investigating the role of virtual surgery and correlations between patient symptoms and CFD-calculated parameters using patients recruited from the Facial Plastic and Reconstructive Surgery clinic at the Medical College of Wisconsin. For this study, a 28 year old asymptomatic female with healthy nasal mucosa was selected. As part of the research protocol a modified contiguous CT scan in the axial plane of the entire nasal cavity and external nasal soft tissues (6.0-mm increments and $0.313-\mathrm{mm}$ resolution) had been obtained. 


\section{Modeling and Simulation}

A 3D reconstruction of the subject's nasal cavity, excluding the paranasal sinuses, was created from the CT scan using the image analysis software Mimics ${ }^{\mathrm{TM}}$ 14.0 (Materialise, Plymouth, MI). Circular septal perforations, 1.0 and $2.0 \mathrm{~cm}$ in diameter, were virtually created in Mimics $^{\mathrm{TM}}$ in anterior, posterior, and superior locations, resulting in a total of 7 models of the nasal airway: 6 with perforations and 1 without. The sizes selected were based on size criteria from previous studies 2,9 . A prior epidemiological study used a location of 1 $\mathrm{cm}$ or more behind the head of the inferior turbinate as the cutoff for a posterior perforation $^{2}$; however, in that study there were relatively few (8\%) perforations located that far posterior. To allow for greater clinical relevance, as well as our own anecdotal evidence that perforations posterior to the head of the inferior turbinate are often asymptomatic, the head of the inferior turbinate was selected as the cutoff point between anterior and posterior perforations. Superior perforations were created high in the anterior septum near the frontal sinus outflow tract, approximating what might be done in association with a modified Lothrop procedure to address frontal sinus disease (figure 1).

Planar nostril and outlet surfaces were crated and the 3D reconstructed nasal cavity models were meshed with approximately 4 million tetrahedral cells using ICEM-CFD ${ }^{\mathrm{TM}} 11.0$ (ANSYS Inc, Canonsburg, PA). Steady-state inspiratory airflow simulations were conducted using Fluent ${ }^{\mathrm{TM}} 12.0$ (ANSYS Inc, Canonsburg, PA) for airflow rates corresponding to normal breathing at rest. The boundary conditions used to determine the airflow field were identical to those previously used ${ }^{10-12}$ : (1) a "wall" condition (zero velocity, stationary wall assumed) at the airway walls; (2) a "pressure-inlet" condition at the nostrils with gauge pressure to 0; and (3) a "pressure-outlet" condition at the outlet with gauge pressure set to a negative value in Pascals $(\mathrm{Pa})$ that generated a target steady-state flow rate. The target airflow rate was based on twice the patient's minute volume (amount of air exhaled in 1 min) which was estimated from body weight. Additional details on the differential equations, computational algorithms, and air physical properties used can be found in a previous publication ${ }^{13}$. Analysis and visualization of results was conducted using Fluent ${ }^{\mathrm{TM}}$ and the post-processing software Fieldview ${ }^{\mathrm{TM}} 12.0$ (Intelligent Light, Lyndhurst, NJ).

\section{Outcome measures}

CFD analysis was used to determine the airflow allocation and total and unilateral nasal resistances for each of the models, including the model with no perforation for comparison. For the perforation models, resistance and airflow allocation were determined for both the region from the nostrils to the posterior edge of the perforation and the region from the posterior edge of the perforation to the posterior septum on each side. Additionally, maximum velocity, maximum wall shear, peak heat flux, and maximum water vapor flux were determined at the site of the perforation itself.

\section{Results}

When the baseline model without a septal perforation was evaluated, the unilateral nasal resistance was $18 \%$ higher on the right side $(0.143 \mathrm{~Pa} /(\mathrm{ml} / \mathrm{s})$ vs. $0.121 \mathrm{~Pa} /(\mathrm{ml} / \mathrm{s}))$, consistent with a mild rightward septal deviation that was confirmed on imaging. Though the subject was asymptomatic and did not report a side preference in her nasal airway, this was associated with a slight left-sided preference in airflow allocation at baseline (54.2\% vs. $45.8 \%$ ). Of note, the CT scan used in this study did not show findings suggestive of an effect due to nasal cycling. When bilateral nasal resistance was examined in each of the models, there was minimal change in each of the septal perforation models compared to the model without a perforation (table 1). 
There was altered airflow allocation in each of the septal perforation models. In all cases the unilateral nasal resistance was higher on the right in the region from the nostrils to the posterior edge of the perforation, and higher on the left in the region posterior to the perforation. Consequently, in each case the airflow was shifted, with more airflow on the left anteriorly followed by more airflow on the right posterior to the perforation as a result of shunting of air through the perforation (figures 2, 3; videos 1, 2). For each location the alteration was greater with the larger size perforation (table 1).

Examination of the physiologic parameters of interest at the actual perforations revealed that for perforations in anterior locations, an increase in size from $1 \mathrm{~cm}$ to $2 \mathrm{~cm}$ increased maximum air velocity by $59 \%(1.74 \mathrm{~m} / \mathrm{s}$ to $2.76 \mathrm{~m} / \mathrm{s})$ and wall shear by $22 \%(0.981 \mathrm{~Pa}$ to $1.192 \mathrm{~Pa}$ ). There was an inverse relationship seen in the posterior perforations, although the magnitude of the velocity difference was less. In the posterior location, an increase in perforation size from $1 \mathrm{~cm}$ to $2 \mathrm{~cm}$ decreased velocity by $5 \%(3.25 \mathrm{~m} / \mathrm{s}$ to $3.10 \mathrm{~m} / \mathrm{s})$ and wall shear by $29 \%$ (1.402 Pa to $1.088 \mathrm{~Pa}$ ). In both anterior and posterior perforations the pattern for heat flux mimicked that of velocity and wall shear, with greater heat exchange in the larger anterior and smaller posterior perforations, while water vapor flux exhibited an opposite relationship. In the superior perforations the velocity at the perforation was greater with the larger perforation, as was the wall shear, although the magnitude of wall shear was less than in the other locations. Also, there was less wall shear and heat and moisture exchange at superior perforations compared to anterior or posterior ones (table 2). Similar to prior reports ${ }^{7,8}$, the maximum values for these physiologic alterations were located at the posterior margin of the perforations (figure 4).

\section{Discussion}

In this study we have used digital nasal models and CFD technology to investigate the changes in nasal physiology due to the presence of septal perforations of varying size and location. It was demonstrated, consistent with prior CFD studies ${ }^{6}$, that there is shunting of air across septal perforations from the higher resistance side to the lower resistance side, with more shunting occurring in larger perforations. We demonstrated that this effect is consistent for perforations in all studied locations, whereas previously this effect had only been demonstrated in anterior perforations. While crusting and epistaxis are the most common complaints in patients with symptomatic septal perforations, obstruction is reported $39 \%$ of the time ${ }^{2}$. As septal perforations do not directly cause nasal obstruction, this sensation of obstruction is likely a result of altered airflow patterns such as the alteration of airflow allocation demonstrated in our study. Furthermore, while the present study only looked at conditioning parameters at the actual perforation site, this alteration of airflow allocation may also lead to alterations in proper heat and moisture exchange throughout the nasal cavities, which may also contribute to a sensation of obstruction directly or as a consequence of excess crust formation.

A previous model study demonstrated that in a symmetrical nose model with a straight septum there was no flow through the perforation. However, in models with both a deviation and a perforation, the location of the deviation with respect to the perforation was a factor. If the deviation was posterior to the perforation, then flow was altered to the side opposite the deviation, while the opposite effect was seen if the deviation was anterior to the perforation ${ }^{5}$. These findings, in conjunction with our results, suggest that if asymmetric or unilateral obstruction is the predominant complaint in a patient with a septal perforation, efforts aimed at producing more balanced airflow allocation (i.e. correction of co-existing septal deviation, unilateral nasal valve compromise, or asymmetric turbinate hypertrophy), if possible, may be indicated. Further, if successful complete closure of the perforation is 
difficult or not possible, efforts to at least decrease the size of the perforation to decrease the amount of shunting that occurs may be indicated.

We discovered an interesting pattern when examining perforations anterior and posterior to the head of the inferior turbinate. In the anterior location, a larger perforation was associated with greater airflow velocity, wall shear stress, and heat loss, while the inverse was seen with the posterior perforations. The exact etiology of this phenomenon is uncertain; however, potential explanations can be inferred from the data. In the anterior nasal cavity, which is an important area for conditioning inspired air, we suspect that the larger perforation provides greater area for air to interact with the perforation. Further, while turbulence was not directly measured in this study, we presume that the increased shear reflects a conversion of laminar to turbulent airflow at the posterior edge. Posteriorly, it has been previously demonstrated that the airflow patterns around the middle and inferior turbinates are more turbulent in nature ${ }^{8}$. We presume that a smaller perforation in this area has greater alterations in velocity and shear as the airflow that is turbulent to begin with is confined within a smaller area, increasing the amount of interaction between the air and mucosa.

It is interesting to note that in the early $20^{\text {th }}$ century surgical enlargement was advocated as an alternative in cases where surgical closure had low likelihood of success ${ }^{14}$. While we do not advocate this strategy, our results suggest this might mitigate some of the problematic physiologic alterations produced by septal perforations in posterior locations. Furthermore, partial closure ${ }^{3,5,15}$ has more recently have been advocated in cases where complete surgical closure of a symptomatic perforation has a low likelihood of success. Our results suggest this strategy may be more appropriate for larger anterior perforations if complete surgical closure is not possible.

Some advanced rhinologic and skull base procedures involve resections of portions of the nasal septum. Some of these techniques, such as a modified Lothrop procedure, involve resection of portions of the superior septum near the frontal sinus outflow tract. It has been traditionally thought that there is little consequence to patients as a result of this. Our results are in agreement with this commonly held belief, as with the superior perforation models studied the alterations of airflow allocation and resistance were minimal, and the alteration of wall shear and heat and moisture flux were markedly less for the superior perforations than those in anterior or posterior locations.

There are some limitations to this study that should be acknowledged. First, the study used models based on a single subject. This introduces potential for bias and no definitive conclusions can be made until more extensive further studies are done. Furthermore, the study design does not allow direct correlation of our objective findings with patient symptoms as this study was performed exclusively using computer simulations and models. Also, for ease of creating the models and standardizing perforation size, all of our perforations were circular in shape, while in reality some perforations may have a more oblong or elliptical configuration. Furthermore, the circular shape and location chosen may only be a rough approximation of the configuration of a superior septectomy done as part of a modified Lothrop. Nasal cycling could potentially contribute to the asymmetry that was seen in airflow allocation and resistance, and we have seen evidence of this in other subjects' scans. However, the CT scan used in this study did not show findings of asymmetric mucosal thickness of the septum or turbinates that would be consistent with a nasal cycling effect.

In conclusion, we have utilized CFD technology to investigate how size and location of septal perforations may alter nasal physiology in an asymptomatic subject. We found that 
larger perforations cause greater alteration in airflow allocation regardless of location, with shunting of air from the higher resistance nasal cavity to the side with lower resistance. Larger anterior and smaller posterior perforations exhibited greater alterations in wall shear and velocity at the site of the perforation. There was relatively little alteration of airflow or conditioning parameters in superior septal perforations.

\title{
Supplementary Material
}

Refer to Web version on PubMed Central for supplementary material.

\section{Acknowledgments}

\author{
Financial support:
}

This research was funded by grant R01EB009557 from the National Institutes of Health/National Institute of Biomedical Imaging and Bioengineering to the Medical College of Wisconsin (MCW) and by subcontract from MCW to the University of North Carolina at Chapel Hill.

\section{References}

1. Oberg D, Akerlund A, Johansson L, Bende M. Prevalence of nasal septal perforation: The skovde population-based study. Rhinology. 2003; 41(2):72-75. [PubMed: 12868370]

2. Diamantopoulos II, Jones NS. The investigation of nasal septal perforations and ulcers. J Laryngol Otol. 2001; 115(7):541-544. [PubMed: 11485583]

3. Brain DJ. Septo-rhinoplasty: The closure of septal perforations. J Laryngol Otol. 1980; 94(5):495505. [PubMed: 7400678]

4. Lanier B, Kai G, Marple B, Wall GM. Pathophysiology and progression of nasal septal perforation. Ann Allergy Asthma Immunol. 2007; 99(6):473-9. quiz 480-1, 521. [PubMed: 18219827]

5. Grutzenmacher S, Mlynski R, Lang C, Scholz S, Saadi R, Mlynski G. The nasal airflow in noses with septal perforation: A model study. ORL J Otorhinolaryngol Relat Spec. 2005; 67(3):142-147. [PubMed: 15925910]

6. Lee HP, Garlapati RR, Chong VF, Wang DY. Effects of septal perforation on nasal airflow: Computer simulation study. J Laryngol Otol. 2010; 124(1):48-54. [PubMed: 19775487]

7. Grant O, Bailie N, Watterson J, Cole J, Gallagher G, Hanna B. Numerical model of a nasal septal perforation. Stud Health Technol Inform. 2004; 107(Pt 2):1352-1356. [PubMed: 15361035]

8. Pless D, Keck T, Wiesmiller KM, Lamche R, Aschoff AJ, Lindemann J. Numerical simulation of airflow patterns and air temperature distribution during inspiration in a nose model with septal perforation. Am J Rhinol. 2004; 18(6):357-362. [PubMed: 15706981]

9. Heller JB, Gabbay JS, Trussler A, Heller MM, Bradley JP. Repair of large nasal septal perforations using facial artery musculomucosal (FAMM) flap. Ann Plast Surg. 2005; 55(5):456-459. [PubMed: 16258293]

10. Kimbell JS, Garcia GJ, Frank DO, Cannon DE, Pawar SS, Rhee JS. Computed nasal resistance compared with patient-reported symptoms in surgically treated nasal airway passages: A preliminary report. Am J Rhinol Allergy. 2012; 26(3):e94-8. [PubMed: 22643935]

11. Rhee JS, Cannon DE, Frank DO, Kimbell JS. Role of virtual surgery in preoperative planning: Assessing the individual components of functional nasal airway surgery. Arch Facial Plast Surg. 2012; 14(5):354-359. [PubMed: 22508896]

12. Frank DO, Kimbell JS, Pawar S, Rhee JS. Effects of anatomy and particle size on nasal sprays and nebulizers. Otolaryngol Head Neck Surg. 2012; 146(2):313-319.

13. Garcia GJ, Bailie N, Martins DA, Kimbell JS. Atrophic rhinitis: A CFD study of air conditioning in the nasal cavity. J Appl Physiol. 2007; 103(3):1082-1092. [PubMed: 17569762]

14. Younger R, Blokmanis A. Nasal septal perforations. J Otolaryngol. 1985; 14(2):125-131. [PubMed: 4068101] 
15. Kridel RW. Considerations in the etiology, treatment, and repair of septal perforations. Facial Plast Surg Clin North Am. 2004; 12(4):435-50. [PubMed: 15337112] 

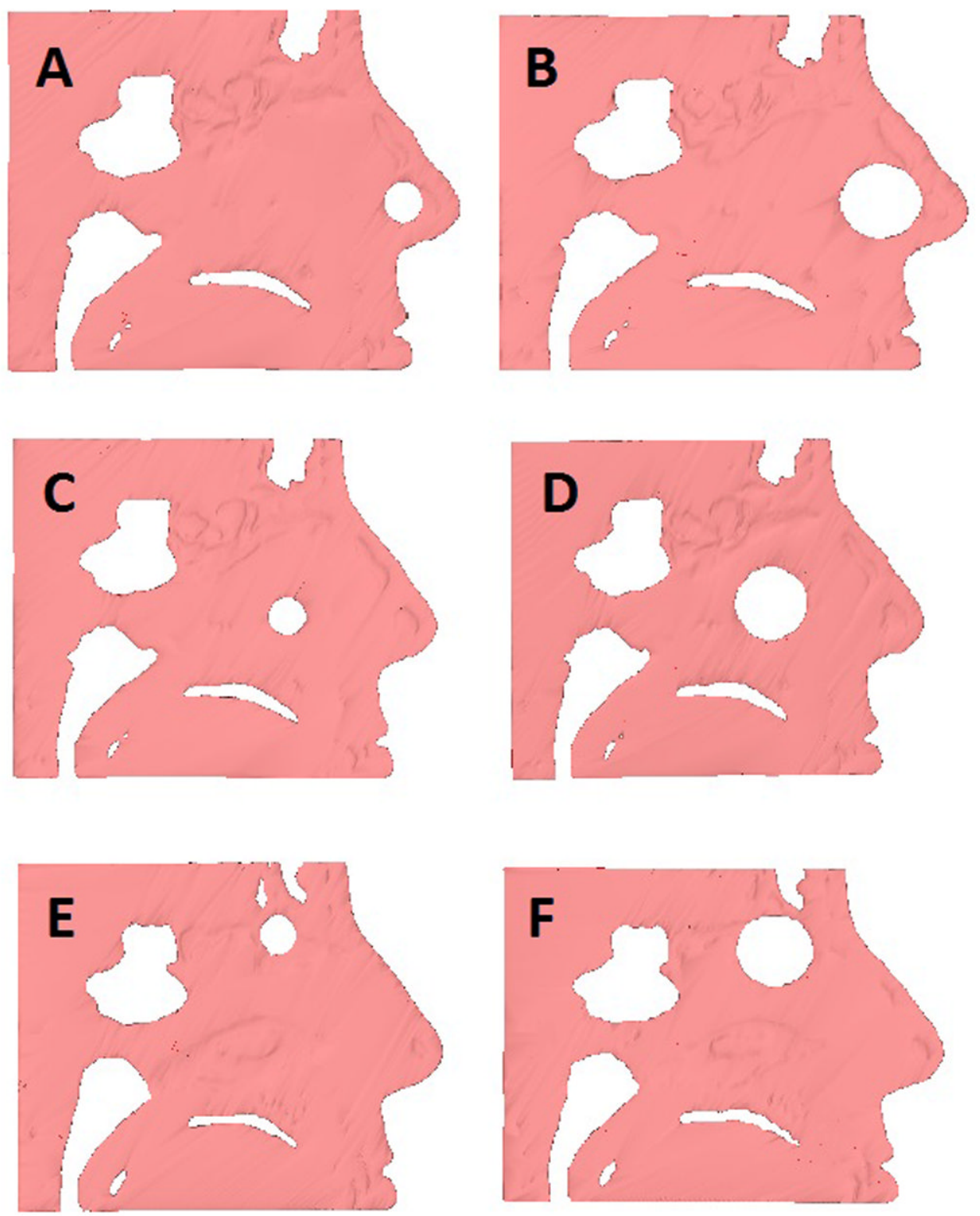

Figure 1.

Cross-sectional sagittal images showing sizes and locations of the modeled perforations. 1 $\mathrm{cm}$ (A) and $2 \mathrm{~cm}(\mathrm{~B})$ anterior perforations, $1 \mathrm{~cm}(\mathrm{C})$ and $2 \mathrm{~cm}$ (D) posterior perforations, and $1 \mathrm{~cm}(\mathrm{E})$ and $2 \mathrm{~cm}(\mathrm{~F})$ superior perforations. 

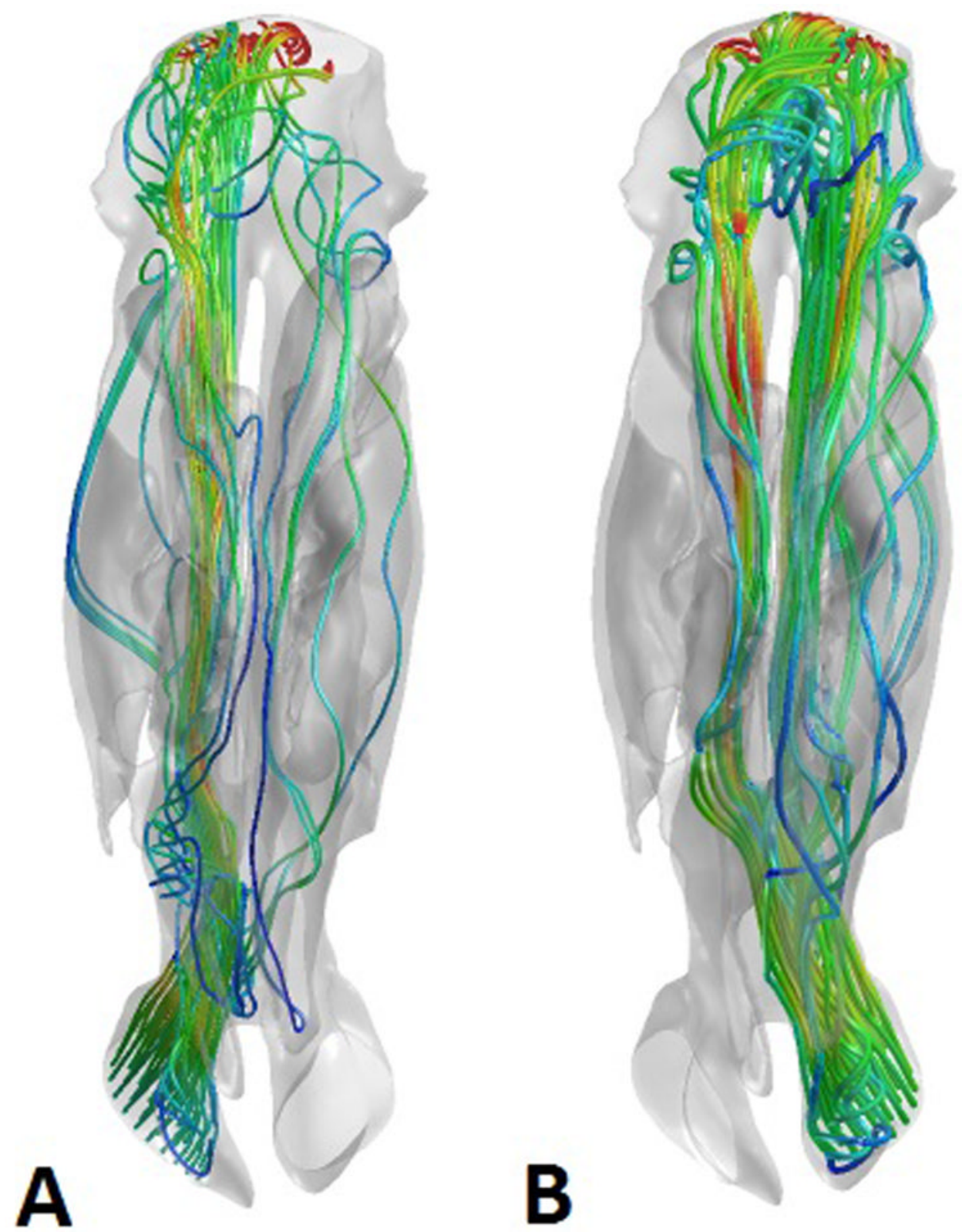

Figure 2.

Representative views of the airstreams through the right (A) and left (B) sides of the nasal cavity in the $2 \mathrm{~cm}$ anterior perforation model, demonstrating predominantly left-to-right shunting of air through the septal perforation. The nasal cavities are viewed from the superior aspect, with the nostrils on the bottom of the figure and the nasopharynx on the top. The airstreams are color coded for velocity, with blue representing low velocity and red representing high velocity. 


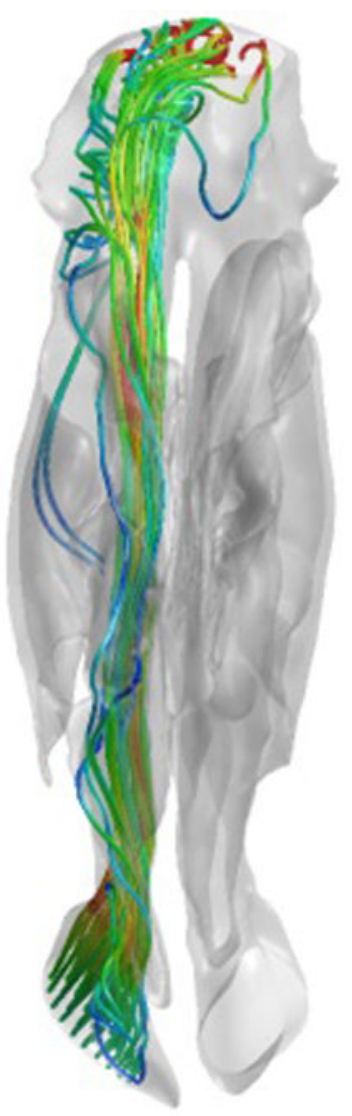

A

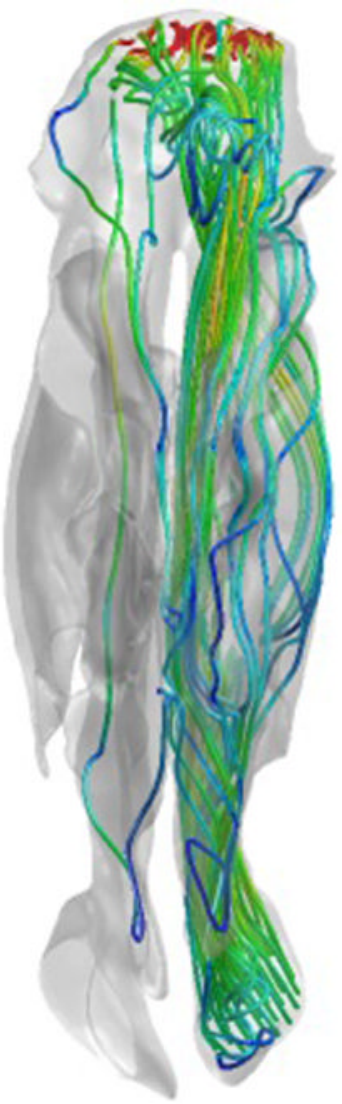

B

Figure 3.

Representative views of the airstreams through the right (A) and left (B) sides of the nasal cavity in the $1 \mathrm{~cm}$ superior perforation model, demonstrating negligible shunting of air through the septal perforation. The nasal cavities are viewed from the superior aspect, with the nostrils on the bottom of the figure and the nasopharynx on the top. The airstreams are color coded for velocity, with blue representing low velocity and red representing high velocity. 

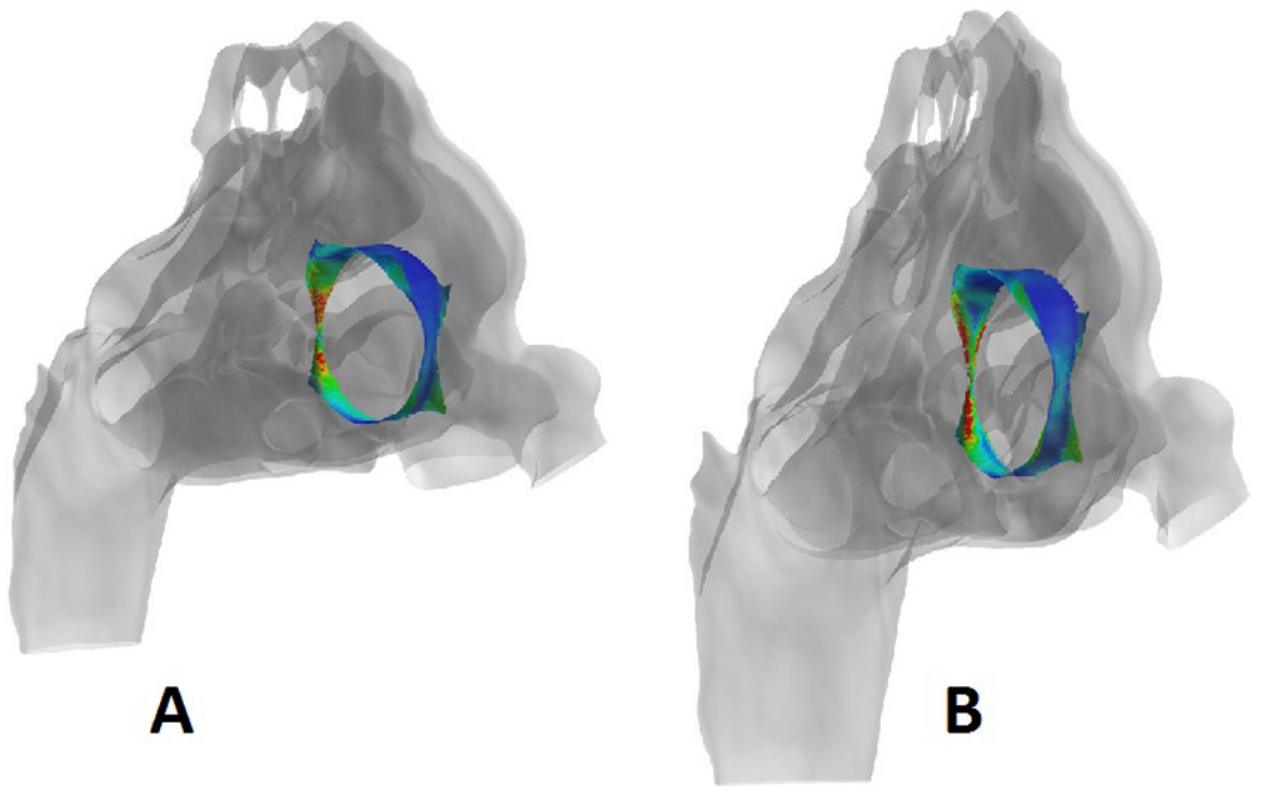

Figure 4.

Representative images from the $2 \mathrm{~cm}$ posterior perforation model showing heat flux (A) and wall shear (B). In each case, the perforation site is color coded with blue representing low values and red high values for the parameter of interest. The perforation has been isolated and the remainder of the nasal cavity rendered transparent to allow visualization and the models are being viewed from an anterior oblique angle, with the nostrils on the right and the nasopharynx on the left. 


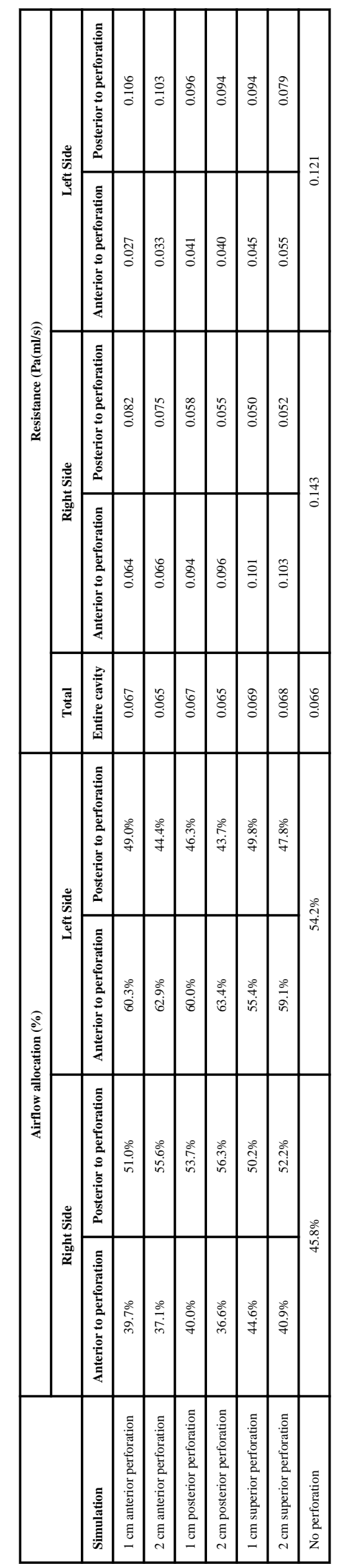


Table 2

CFD-calculated parameters occurring at the perforation site

\begin{tabular}{|l|c|c|c|c|}
\hline Simulation & Maximum velocity $(\mathbf{m} / \mathbf{s})$ & $\begin{array}{c}\text { Maximum wall shear } \\
(\mathbf{P a})\end{array}$ & Peak heat flux (W/m2) & $\begin{array}{c}\text { Maximum water vapor } \\
\text { flux (kg(s-m2)) }\end{array}$ \\
\hline $1 \mathrm{~cm}$ anterior perforation & 1.738 & 0.981 & 3081.781 & 0.0046 \\
\hline $2 \mathrm{~cm}$ anterior perforation & 2.758 & 1.192 & 3403.828 & 0.0039 \\
\hline $1 \mathrm{~cm}$ posterior perforation & 3.249 & 1.402 & 4275.234 & 0.0037 \\
\hline $2 \mathrm{~cm}$ posterior perforation & 3.098 & 1.088 & 3835.188 & 0.0040 \\
\hline $1 \mathrm{~cm}$ superior perforation & 1.815 & 0.380 & 632.734 & 0.0005 \\
\hline $2 \mathrm{~cm}$ superior perforation & 2.023 & 0.482 & 838.625 & 0.0008 \\
\hline
\end{tabular}

